\title{
POLYNOMIAL APPROXIMATION ON COMPACT MANIFOLDS AND HOMOGENEOUS SPACES
}

\author{
BY \\ DAVID L. RAGOZIN( $\left.{ }^{1}\right)$
}

\begin{abstract}
We prove several theorems which relate the smoothness of a function, $f$, defined on a compact $C^{\infty}$-submanifold of a Euclidean space to the rate at which the error in the best uniform approximation to $f$ by polynomials of degree at most $n$ tends to zero.
\end{abstract}

Introduction. In this paper we extend the classical Jackson and Bernstein theorems on uniform polynomial approximation to a wide class of compact $C^{\infty}$-manifolds. $\S 1$ contains the definitions, for an arbitrary compact manifold $M$ of the Banach spaces $C^{k, \omega}(M)$ of $k$-times differentiable functions whose $k$ th derivatives admit $\omega$ as a modulus of continuity, and the proof of a Tietze type extension theorem for $C^{k, \omega}$ functions. In $\S 2$ we consider manifolds $M$ embedded in some Euclidean space $\boldsymbol{E}$ and prove the Jackson estimate that for $f \in C^{k, \omega}(M)$, $E_{n}(f)=O\left(n^{-k} \omega(1 / n)\right)$, where $E_{n}(f)$ is the error in the best uniform approximation to $f$ on $M$ by polynomials of degree at most $n$. In $\S 3$, as a preliminary to considering converses of the Jackson estimates, we determine the asymptotic behaviour of the widths of the unit ball in $C^{k, \omega}(M)$ and use this to show that unless $M^{m}$ is embedded in $\boldsymbol{E}$ as a subset of an algebraic variety of dimension $m$ no converse results can hold. $\S \S 4$ and 5 are restricted to the case when $M$ is the orbit of some orthogonal action of a compact Lie group on $\boldsymbol{E}$. In this case we prove a Bernstein type inequality for the derivatives of polynomials on $M$. This is applied in $\S 5$ to prove several converse theorems, including the Bernstein theorems which say $E_{n}(f)$ $=O\left(n^{-k-\alpha}\right), 0<\alpha<1$, implies $f \in C^{k}(M)$ and all $k$ th derivatives of $f$ satisfy a Lipschitz condition of order $\alpha$.

1. Classes of functions. In this section we give the definitions of the Banach spaces of smooth functions $C^{k, \omega}(M)$ where $M$ is a compact $C^{\infty}$-manifold (perhaps with boundary) and $\omega$ is a modulus of continuity (see below for a definition). These spaces are slightly more general than the $C^{k,(\alpha)}(M)$ spaces of $k$-times

Received by the editors August 30, 1968.

AMS Subject Classifications. Primary 2270, 4115, 4141, 5750.

Key Words and Phrases. Polynomial approximation, Jackson theorems, $n$-width, function spaces on manifolds, differential operators, homogeneous spaces, Bernstein's inequality.

(1) Many of the results of this paper are contained in the author's Ph.D. thesis written at Harvard University under the direction of Professor A. Gleason, whom the author would like to thank. The writing of this paper was supported in part by NSF Grants GP-9003 and GP-7461. 
differentiable functions with $k$ th derivatives satisfying a Lipschitz (Hölder) condition of order $\alpha$. We also prove an extension theorem for $C^{k, \omega}$ functions on a closed submanifold.

We start by recalling the definition of a modulus of continuity. This is a continuous function $\omega: \boldsymbol{R}^{+} \rightarrow \boldsymbol{R}^{+}$satisfying

(i) $\omega$ is increasing,

(ii) $\omega(t) \rightarrow 0$ as $t \rightarrow 0$,

(iii) $\omega\left(t_{1}+t_{2}\right) \leqq \omega\left(t_{1}\right)+\omega\left(t_{2}\right)$,

(iv) for some $a>0, t \leqq a \omega(t), 0<t \leqq 2$.

The most important examples are the functions $\omega_{\alpha}(t)=t^{\alpha}, 0<\alpha \leqq 1$.

Let $B^{m}$ be the unit ball in Euclidean $m$-space. If $f$ is a continuous complexvalued function on $B^{m}$ and $\omega$ is as above, we say $f$ admits $\omega$ as a modulus of continuity in case

$$
|f|_{\omega}=\sup _{x \neq y} \frac{|f(x)-f(y)|}{\omega(\|x-y\|)}
$$

is finite. The class of all such functions, denoted by $C^{0, \omega}\left(B^{m}\right)$, is a Banach space under the norm $\|f\|_{0, \omega}=\|f\|_{\infty}+|f|_{\omega}$.

The class of $k$-times differentiable functions on $B^{m}$ with $k$ th derivatives admitting $\omega$ as a modulus of continuity will be denoted by $C^{k, \omega}\left(B^{m}\right)$. It is a Banach space under the norm

$$
\|f\|_{k, \omega}=\sum_{|\beta| \leqq k}\left\|\frac{\partial^{\beta} f}{\partial x^{\beta}}\right\|_{\infty}+\sum_{|\beta|=k}\left|\frac{\partial^{\beta} f}{\partial x^{\beta}}\right|_{\omega}
$$

where $\beta$ is an $m$-dimensional multi-index. We shall write $\|f\|_{k}$ for the first summand in the above expression.

We note that the following are continuous linear transformations of $C^{k, \omega}\left(B^{m}\right)$ into itself: (a) multiplication by $C^{\infty}$-functions, (b) $f \mapsto f \circ \varphi$ where $\varphi: B^{m} \rightarrow B^{m}$ is any $C^{\infty}$-map. It is straightforward to verify these facts once we observe that property (iv) of $\omega$ shows that $C^{1}\left(B^{m}\right) \subseteq C^{0, \omega}\left(B^{m}\right)$, since

for $f$ in $C^{1}\left(B^{m}\right)$.

$$
|f(x)-f(y)| \leqq \sum\left\|\frac{\partial f}{\partial x_{i}}\right\|_{\infty}\|x-y\| \leqq\|f\|_{1} a \omega(\|x-y\|)
$$

We extend our definitions to an arbitrary compact $C^{\infty}$-manifold $M$ of dimension $m$ as follows,

$$
C^{k, \omega}(M)=\left\{\begin{array}{l|l}
f \in C^{k}(M) & \begin{array}{l}
\text { For each } x \in M, \text { there exists a chart } \\
\varphi: B^{m} \rightarrow M \text { with } x \in \operatorname{Int} \varphi\left(B^{m}\right) \text { and } \\
f \circ \varphi \in C^{k, \omega}\left(B^{m}\right)
\end{array}
\end{array}\right\} .
$$

The remarks of the previous paragraph make it possible to show that if

$$
\varphi_{i}: B^{m} \rightarrow M, \quad i=1, \ldots, l,
$$

are a finite collection of charts with $M=\bigcup \operatorname{Int} \varphi_{i}\left(B^{m}\right)$, then $f \in C^{k, \omega}(M)$ if and only if $f \circ \varphi_{i} \in C^{k, \omega}\left(B^{m}\right)$ for each $i$. Moreover, the norm $\|f\|_{k, \omega}=\sum\left\|f \circ \varphi_{i}\right\|_{k, \omega}$ is a 
Banach space norm for $C^{k, \omega}(M)$ and any other choice of finitely many charts covering $M$ gives an equivalent norm. In the special case when $\omega=\omega_{\alpha}, 0<\alpha \leqq 1$, we shall write $C^{k,(\alpha)}(M)$ instead of $C^{k, \omega_{\alpha}}(M)$.

The following analog of the Tietze extension theorem forms the basis of our proof of the Jackson estimates in $\S 2$.

THEOREM 1.1. If $N^{n}$ is a compact manifold embedded in $M \backslash \partial M$, then there exists a continuous linear extension map e: $C^{k, \omega}(N) \rightarrow C^{k, \omega}(M)$.

Proof. We begin by solving the local extension problem and then extend globally via a partition of unity. There are two local problems, extension from a ball of lower dimension and extension from the upper half-ball. For the first, let $B^{n}=\pi\left(B^{m}\right)$ where $\pi: B^{m} \rightarrow B^{m}$ is the projection on the first $n$ coordinates. Then the map $\pi^{*}: C^{k, \omega}\left(B^{n}\right) \rightarrow C^{k, \omega}\left(B^{m}\right)$ given by $\pi^{*} f=f \circ \pi$ is a continuous linear extension since $\pi$ is a $C^{\infty}$-map and $\pi=$ identity on $B^{n}$. For the second local problem let $B_{+}^{n}=\left\{x \in B^{n} \mid x_{1} \geqq 0\right\}$ and write $x \in B^{n}$ as $x=\left(x_{1}, x^{*}\right)$. Then the extension map $e_{k}$ defined by

$$
\begin{array}{rlrl}
e_{k}(f)(x) & =f\left(x_{1}, x^{*}\right), & x_{1} \geqq 0, \\
& =\sum_{i=0}^{k} C_{i} f\left(-2^{-i} x_{1}, x^{*}\right), & & x_{1}<0,
\end{array}
$$

where the $C_{i}$ are chosen to satisfy $\sum_{0}^{k} C_{i}\left(-2^{-i}\right)^{j}=1, j=0,1, \ldots, k$, is a continuous map from $C^{k, \omega}\left(B_{+}^{n}\right)$ to $C^{k, \omega}\left(B^{n}\right)$. That $e_{k}$ takes $C^{k}\left(B_{+}^{n}\right)$ to $C^{k}\left(B^{n}\right)$ is easily verified since the $C_{i}$ have been chosen so as to make all derivatives of $e_{k}(f)$ of order at most $k$ continuous across the set where $x_{1}=0$. The fact that $\partial^{\beta} e_{k}(f) / \partial x^{\beta},|\beta|=k$, admits $\omega$ as a modulus of continuity follows from the fact that $\partial^{\beta} f / \partial x^{\beta}$ does.

As a preliminary to patching together these local results, we note that if $\varphi: B^{m} \rightarrow M$ is any chart for $M$ and $h \in C^{\infty}(M)$ has supp $(h) \subseteq \operatorname{Int} \varphi\left(B^{m}\right)$, then the linear map $h^{\#:} C^{k, \omega}\left(B^{m}\right) \rightarrow C^{k, \omega}(M)$, defined by

$$
\begin{aligned}
h^{\#}(f)(m) & =0, & & m \in M \backslash \varphi\left(B^{m}\right), \\
& =h(m) f\left(\varphi^{-1}(m)\right), & & m \in \varphi\left(B^{m}\right),
\end{aligned}
$$

is continuous. That $h^{\#}(f) \in C^{k, \omega}(M)$ follows by the definition of this class since $h^{\#}(f) \equiv 0$ outside $\operatorname{supp}(h) \subseteq \operatorname{Int} \varphi\left(B^{m}\right)$. Moreover if we norm $C^{k, \omega}(M)$ using $\varphi$ and other charts with images outside supp $(h)$, then $\left\|h^{\#}(f)\right\|_{k, \omega}=\|(h \circ \varphi) f\|_{k, \omega}$.

Finally, since $N \subseteq M \backslash \partial M$, we can find charts $\varphi_{i}: B^{m} \rightarrow M, i=1, \ldots, l$, with $N \subseteq \bigcup$ Int $\varphi_{i}\left(B^{m}\right)$ and $\partial M \cap \varphi_{i}\left(B^{m}\right)=\varnothing$, and such that for $i=1, \ldots, j, \varphi_{i}^{-1}(N)$ $\cap B^{m}=B^{n}$, while for $i=j+1, \ldots, l, \varphi_{i}^{-1}(N) \cap B^{m}=B_{+}^{n}$. Let $h_{i} \in C^{\infty}(M)$ be chosen with supp $\left(h_{i}\right) \subseteq \operatorname{Int} \varphi_{i}\left(B^{m}\right)$ and $\sum h_{i}=1$ on $N$. Then the desired extension map is given, for $f \in C^{k, \omega}(N)$, by

$$
e(f)=\sum_{1}^{j} h_{i}^{\#} \pi^{*}\left(f \circ \varphi_{i}\right)+\sum_{j+1}^{l} h_{i}^{\#} \pi^{*} e_{k}\left(f \circ \varphi_{i}\right) .
$$

That $e$ does the job follows from the facts we have proved about $h_{i}^{\#}, \pi^{*}$, and $e_{k}$. 
2. Direct approximation theorems. Let $\boldsymbol{E}$ be a Euclidean space and $M \subseteq E$ a compact $C^{\infty}$-submanifold. We set

$$
\mathscr{P}_{n}=\left\{p \in C(M) \mid \begin{array}{c}
p=p_{n}\left\lceil M \text { for some polynomial } p_{n} \in C(\boldsymbol{E})\right. \\
\text { of (total) degree at most } n
\end{array}\right\}
$$

and refer to $\mathscr{P}_{n}$ as the polynomials of degree at most $n$ on $M$. A measure of the degree of uniform approximation to $f \in C(M)$ by $\mathscr{P}_{n}$ is given by

$$
E_{n}(f)=\inf \left\{\left\|f-p_{n}\right\|_{\infty} \mid p_{n} \in \mathscr{P}_{n}\right\} .
$$

Since $\mathscr{P}_{n}$ is finite dimensional this infimum is attained for at least one $p_{n}$ in $\mathscr{P}_{n}$. Thus we can speak of $E_{n}(f)$ as the error in the best uniform approximation to $f$ by polynomials of degree at most $n$.

The Stone-Weierstrass theorem implies that $E_{n}(f) \rightarrow 0$ as $n \rightarrow \infty$. For the special case when $M$ is a cube or a multi-dimensional torus, the multi-dimensional version of the theorems of Jackson [5, pp. 89-90] give a more precise relationship between the smoothness of $f$ and the rate at which $E_{n}(f)$ tends to zero. The main result of this section is the extension of the Jackson theorems to the present setting.

THEOREM 2.1. Let $M \subseteq E$ be a compact $C^{\infty}$-submanifold. Fix a norm on $C^{k, \omega}(M)$. Then there exist constants $C(k, \omega)$ such that

$$
f \in C^{k, \omega}(M) \text { implies } E_{n}(f) \leqq C(k, \omega)\|f\|_{k, \omega} n^{-k} \omega(1 / n) .
$$

Proof. The extension theorem together with the Jackson theorems for a ball (or cube) make this easy. We may assume that $M \subseteq \operatorname{Int} B^{l}$, where $l=\operatorname{dim} E$. Then, by the continuity of the extension map $e$ of Theorem 1.1 , there exist constants $A(k, \omega)$ such that $\|e(f)\|_{k, \omega} \leqq A(k, \omega)\|f\|_{k, \omega}$. The Jackson theorems for $B^{l}$ [12] imply the existence of constants $A(k)$ and a sequence $\left\{p_{n}\right\}$ of polynomials on $B^{l}$ with $p_{n}$ of degree at most $n$ such that

$$
\left\|e(f)-p_{n}\right\|_{\infty, B} \leqq A(k)\|e(f)\|_{k, \omega} n^{-k} \omega(1 / n) .
$$

Restriction of the sequence $\left\{p_{n}\right\}$ to $M$ gives the desired result with $C(k, \omega)=$ $A(k) A(k, \omega)$. (We could have used the Jackson theorems for a cube rather than for $B^{l}$. But this would require a bit more work, since for a cube they are stated in terms of polynomials of degree at most $n$ in each variable separately, rather than total degree at most $n$.)

We single out the special case of functions with $k$ th derivatives satisfying a Lipschitz condition of order $\alpha$ to state as

COROLlaRy 2.2. For $0<\alpha \leqq 1$, there exists $C(k, \alpha)$ such that

$$
f \in C^{k,(\alpha)}(M) \text { implies } E_{n}(f) \leqq C(k, \alpha)\|f\|_{k,(\alpha)} n^{-k-\alpha} \text {. }
$$

Whether Theorem 2.1 gives good estimates for the rate of decrease of $E_{n}(f)$ seems to depend on the nature of the embedding of $M$. Thus for $S^{1} \subseteq E^{2}$ the Bern- 
stein converse theorems show that in case $\omega=\omega_{\alpha}, 0<\alpha<1$, then the estimates cannot be improved in the sense that there exist $f \in C^{k,(\alpha)}\left(S^{1}\right)$ for which $E_{n}(f)$ $\geqq C n^{-k-\alpha}$. On the other hand, Newman and Raymon in [8], give examples of analytic arcs $\gamma \subseteq E^{2}$ for which $f \in C^{0, \omega}(\gamma)$ implies $E_{n}(f) \leqq C|f|_{\omega} \omega\left(n^{-2}\right)$, which shows that Theorem 1.1 gives far from good estimates for these curves. In the next section we shall show that by perturbing the embedding of $M$ slightly we can insure that the estimates of Theorem 2.1 cannot be improved.

3. Widths. We are interested in finding some class of embedded manifolds for which the converse to Corollary 2.2 holds, at least for $0<\alpha<1$. We know from the classical case of $M=[0,1] \subseteq E^{1}$ that if $\partial M \neq \varnothing$ we cannot expect any such converse to hold since the behaviour of the approximation near $\partial M$ is not taken into account in the estimates of Theorem 2.1. (In fact, whether there is, in general, an improvement in the rate of approximation near $\partial M$ is not known to us.) However, we shall show that even if $\partial M=\varnothing$, the nature of the embedding can rule out the existence of any converse to Theorem 2.1 or Corollary 2.2.

We begin by recalling the notion of the widths of a set $U$ in a Banach space $X$. (See [5, Chapter 9].) If $X_{n}$ is an $n$-dimensional subspace of $X$, then the degree of approximation to $U$ by $X_{n}$ is given by

$$
E_{X_{n}}(U)=\sup _{f \in X} \inf _{p \in X_{n}}\|f-p\| .
$$

The $n$th width of $U$ in $X$ is defined by $d_{n}(U)=\inf _{X_{n}} E_{X_{n}}(U)$. It is useful to notice that $d_{n}$ is increasing as a function of $U$ and also if $r>0$, then $d_{n}(r U)=r d_{n}(U)$.

Theorem 2.1 gives some estimates from above for the widths of the sets $U^{k, \omega}$ $=\left\{f \in C^{k, \omega}(M) \mid\|f\|_{k, \omega} \leqq 1\right\} \subseteq C(M)$. In particular, if we set $\delta(n)=\operatorname{dim} \mathscr{P}_{n}$, then the theorem shows that $d_{\delta(n)}\left(U^{k, \omega}\right)=O\left(n^{-k} \omega(1 / n)\right)$. The next result shows that any converse to Theorem 2.1 implies corresponding estimates from below for the widths.

THEOREM 3.1. Suppose that for some $k$ and $\omega$ there exists a constant $B$ such that for $f \in C(M)$

$$
E_{n}(f) \leqq n^{-k} \omega(1 / n) \quad \text { implies } f \in B U^{k, \omega} .
$$

Then $n^{-k} \omega(1 / n)=O\left(d_{\delta(n)}\left(U^{k, \omega}\right)\right)$.

Proof. Let $U=\left\{f \in C(M) \mid E_{n}(f) \leqq n^{-k} \omega(1 / n)\right\}$. Then by Lorentz's theorem on full approximation sets [5, p. 139], ${ }^{-} d_{\delta(n)}(U)=n^{-k} \omega(1 / n)$. If (3.1.1) holds, then $U \subseteq B U^{k, \omega}$. Thus

$$
n^{-k} \omega(1 / n)=d_{\delta(n)}(U) \leqq d_{\delta(n)}\left(B U^{k, \omega}\right)=B d_{\delta(n)}\left(U^{k, \omega}\right)
$$

as desired.

Since the widths, $d_{n}\left(U^{k, \omega}\right)$, are intrinsic to the space $C(M)$, while the dimension function $\delta(n)$ depends on the embedding of $M$ in $E$ we see that a determination of 
the asymptotic behaviour of these widths will give information as to which embeddings admit the possibility of any converse implication of the form (3.1.1). We proceed to find the desired asymptotic estimates. Our method is to localize and show that everything is determined by the widths for the case $M=B^{m}$.

THEOREM 3.2. Let $M$ be a compact $C^{\infty}$-manifold of dimension $m$. Fix a norm on $C^{k, \omega}(M)$ and let $U^{k, \omega}$ be the unit ball in this norm. Then $\left({ }^{2}\right) d_{n}\left(U^{k, \omega}\right) \approx n^{-k / m} \omega\left(n^{-1 / m}\right)$.

Proof. First we show that $d_{n}\left(U^{k, \omega}\right)=O\left(n^{-k / m} \omega\left(n^{-1 / m}\right)\right)$. Let $\varphi_{i}: B^{m} \rightarrow M$, $i=1, \ldots, l$, be the charts for $M$ with $M=\bigcup \operatorname{Int} \varphi_{i}\left(B^{m}\right)$ which define the norm for $C^{k, \omega}(M)$ and let $\left\{h_{i}\right\}$ be a partition of unity with supp $\left(h_{i}\right) \subseteq \operatorname{Int} \varphi_{i}\left(B^{m}\right)$. If $\boldsymbol{P}_{n}$ is the space of polynomials on $B^{m}$ of degree at most $n$, then for $f \in C^{k, \omega}(M)$ and each $i$ the Jackson theorems for $B^{m}$ show there exists a constant $C(k, \omega)$ and a sequence of polynomials $\left\{p_{n, i}\right\}, p_{n, i} \in \boldsymbol{P}_{n}$, such that

Let

$$
\left\|f \circ \varphi_{i}-p_{n, i}\right\|_{\infty, B^{m}} \leqq C(k, \omega)\left\|f \circ \varphi_{i}\right\|_{k, \omega} n^{-k} \omega(1 / n) .
$$

$$
Q_{n}=h_{1}^{\#}\left(\boldsymbol{P}_{n}\right)+h_{2}^{\#}\left(\boldsymbol{P}_{n}\right)+\cdots+h_{l}^{\#}\left(\boldsymbol{P}_{n}\right),
$$

where for $p \in C\left(B^{m}\right), h_{i}^{\#}(p)(m)=h_{i}(m) p\left(\varphi_{i}^{-1}(m)\right)$. Then $Q_{n}$ is a subspace of $C(M)$ with $\operatorname{dim} Q_{n}=q(n) \approx n^{m}$. Now for $f \in U^{k, \omega}$ we have, since $f=\sum h_{i} f,\left\|h_{i}\right\|_{\infty} \leqq 1$ and $\sum\left\|f \circ \varphi_{i}\right\|_{k, \omega} \leqq 1$,

$$
\begin{aligned}
\left\|f-\sum h_{i}^{\#}\left(p_{n, i}\right)\right\|_{\infty, M} & \leqq \sum\left\|\left(h_{i} f\right) \circ \varphi_{i}-\left(h_{i} \circ \varphi_{i}\right) p_{n, i}\right\|_{\infty, B^{m}} \\
& \leqq \sum\left\|f \circ \varphi_{i}-p_{n, i}\right\|_{\infty, B^{m}} \\
& \leqq C(k, \omega) n^{-k} \omega(1 / n) .
\end{aligned}
$$

Thus,

$$
d_{q(n)}\left(U^{k, \omega}\right) \leqq E_{Q_{n}}\left(U^{k, \omega}\right)=O\left(q(n)^{-k / m} \omega\left(q(n)^{-1 / m}\right)\right) .
$$

This yields $d_{n}\left(U^{k, \omega}\right)=O\left(n^{-k / m} \omega\left(n^{-1 / m}\right)\right)$ since $q(n+1) / q(n)$ is bounded.

Next we show that $d_{n}\left(U^{k, \omega}\right) \geqq C n^{-k / m} \omega\left(n^{-1 / m}\right)$ by using the extension map $e$ of Theorem 1.1 , in case $N=B^{m}$ is embedded in $M$ via a chart $\varphi$. Thus

$$
e: C^{k, \omega}\left(B^{m}\right) \rightarrow C^{k, \omega}(M) \text {. }
$$

If we let $V^{k, \omega}$ be the unit ball in $C^{k, \omega}\left(B^{m}\right)$, then by the continuity of $e$, we have $e\left(V^{k, \omega}\right) \subseteq C(k, \omega) U^{k, \omega}$ for some constant $C(k, \omega)$. So it will suffice to show that $d_{n}\left(e\left(V^{k, \omega}\right)\right) \geqq C n^{-k / m} \omega\left(n^{-1 / m}\right)$.

Let $X_{n} \subseteq C(M)$ be an arbitrary $n$-dimensional subspace. Then for $f \in V^{k, \omega}$, $p \in X_{n},\|e(f)-p\|_{\infty, M} \geqq\|f-p\|_{\infty, B^{m}}$. Hence, taking inf's, $E_{X_{n}}\left(e\left(V^{k, \omega}\right)\right) \geqq d_{n}\left(V^{k, \omega}\right)$, and thus $d_{n}\left(e\left(V^{k, \omega}\right)\right) \geqq d_{n}\left(V^{k, \omega}\right)$. Now [5, Theorem 1, p. 135] shows that $d_{n}\left(V^{k, \omega}\right)$ $\geqq C n^{-k / m} \omega\left(n^{-1 / m}\right)$ and so we are done. (The proof in [5] is for functions on a cube, but holds without essential change for functions on a ball.)

$\left.{ }^{2}\right)$ By $\approx$ we mean that each sequence is big-oh of the other. 
Corollary 3.3. Let $M \subseteq E$ be a compact submanifold of dimension $m$ and set $\delta(n)=\operatorname{dim} \mathscr{P}_{n}$.

(a) If $\delta(n)=O\left(n^{m}\right)$ then the estimates of Theorem 2.1 cannot be improved as regards order of decrease.

(b) If a converse theorem of the form (3.1.1) is true for $M$, then $\delta(n)=O\left(n^{m}\right)$.

These corollaries show that the order of growth of $\delta(n)$ is an important characteristic of the embedding of $M$ in $E$, so far as the approximation properties of polynomials is concerned. It is, therefore, not surprising that the function $\delta(n)$ is closely connected with the algebraic properties of the given embedding. Specifically, it is a consequence of the affine version of the theorems on the Hilbert polynomial of a graded module [15, pp. 232-236] that for any $M \subseteq E, \delta(n)$ is, for $n$ sufficiently large, a polynomial in $n$ of degree equal to the dimension (in the sense of algebraic geometry) of the smallest algebraic variety in $\boldsymbol{E}$ containing $M$. This dimension is always at least $m$. Hence $\delta(n)=O\left(n^{m}\right)$ if and only if $M$ is contained in an algebraic variety of dimension $m$. In particular, if $M$ itself is an algebraic variety then $\delta(n)=O\left(n^{m}\right)$. (This depends on the fact that then the dimension of $M$ as a real algebraic variety is equal to its dimension as a manifold, which is a consequence of Theorem 1 and Lemma 9 of [14].)

Nash has proved [7, Theorem 1] that when $\partial M=\varnothing$ any embedding of $M$ in $E$ can be approximated by an embedding in which $M$ is included in an algebraic variety of dimension $m$. (Although stated for connected manifolds, Nash's theorem extends by taking unions to the case when $M$ is not connected.) Thus by perturbing the embedding we can assure that the hypothesis of Corollary 3.3a is satisfied.

For embeddings of $M$ with $\delta(n) \neq O\left(n^{m}\right)$ we do not know whether the estimates of Theorem 2.1 can always be improved. In the previously cited example of Newman and Raymon of a curve $\gamma \subseteq E^{2}$ for which $\delta(n)=O\left(n^{2}\right)$, their better estimates show that $E_{n}(f)=O\left(d_{\delta(n)}\left(U^{0, \omega}\right)\right)$ uniformly for $f \in U^{0, \omega}$. It is an interesting question whether this improvement holds for any $M \subseteq E$, i.e. is it always the case that $E_{n}(f)=O\left(d_{\delta(n)}\left(U^{k, \omega}\right)\right)$ uniformly for $f \in U^{k, \omega}$ ?

Another question which is open, so far as we know, is whether the converse of Corollary 3.3b holds, i.e. does every manifold $M \subseteq E$ with $\partial M=\varnothing$ which is (included in) an algebraic variety of dimension $m$ satisfy some converse theorem of the form (3.1.1)? In the next two sections we show that such theorems do hold for the special case of a homogeneous space of a compact Lie group equivariantly embedded in $\boldsymbol{E}$.

4. Compact homogeneous spaces and Bernstein's inequality. Let $M=G / H$ be a homogeneous space of the compact Lie group $G$. Then it is known [6, p. 433] that we may embed $M$ in some Euclidean space $E$ as the orbit of an orthogonal action of $G$ on $E$. By passing to the quotient of $G$ by the kernel of this orthogonal representation, we may assume that $G$ is a closed subgroup of $O(E)$, the orthogonal 
group of $\boldsymbol{E}$, and that $M=G v$ is the $G$-orbit of some unit vector, $v$, in $\boldsymbol{E}$. Then $H=\{g \in G \mid g \cdot v=v\}$.

The action of $G$ on $M$ induces an action of $G$ on $C(M)$ according to $g \cdot f(m)$ $=f\left(g^{-1} \cdot m\right), f \in C(M)$. This action leaves the classes $\mathscr{P}_{n}$ invariant. To see this we let $e_{1}=v, e_{2}, \ldots, e_{l}$ be an orthonormal basis for $\boldsymbol{E}$. Then the coordinate functions, $f_{j}$, on $M$ are given by $f_{j}(m)=\left(m, e_{j}\right)$, where $(\cdot, \cdot)$ is the inner product on $\boldsymbol{E}$, and $\mathscr{P}_{n}$ consists of polynomials in $f_{1}, \ldots, f_{l}$ of degree at most $n$. Now if the matrix of $g=\left(g_{\imath j}\right)$ relative to the basis $\left\{e_{i}\right\}$, then

$$
\begin{aligned}
g \cdot f_{j}(m) & =f_{j}\left(g^{-1} \cdot m\right)=\left(g^{-1} \cdot m, e_{j}\right)=\left(m, g \cdot e_{j}\right) \\
& =\sum\left(m, g_{i j} e_{i}\right)=\sum g_{i j} f_{i}(m) .
\end{aligned}
$$

Or, $g \cdot f_{j}=\sum g_{i j} f_{i}$. So $\mathscr{P}_{1}$ is $G$-invariant. Hence $\mathscr{P}_{n}$ is $G$-invariant since it consists of $n$th degree polynomials in the elements of $\mathscr{P}_{1}$.

We are interested in the norms of certain differential operators on the spaces $\mathscr{P}_{n}$. These are the operators that arise from the Lie algebra, $\mathfrak{g}$, of $G$, where $\mathfrak{g}$ consists of those $l \times l$ skew-symmetric matrices $D$ such that $\exp t D \in G$ for all $t \in \boldsymbol{R}$. Each $D \in \mathrm{g}$ acts as a differential operator on $C^{1}(M)$ by

$$
D f(m)=(d / d t) f(\exp t D \cdot m)_{t=0}, \quad f \in C^{1}(M) .
$$

Note that each $D \in \mathrm{g}$ leaves $\mathscr{P}_{n}$ invariant since $D p_{n}$ is the uniform limit as $t \rightarrow 0$ of the functions $\left(\exp (-t D) \cdot p_{n}-p_{n}\right) / t$ which are in $\mathscr{P}_{n}$ and $\mathscr{P}_{n}$ is uniformly closed. We will use the inner product on $g$ given by

$$
\left\langle D_{1}, D_{2}\right\rangle=\frac{1}{2} \operatorname{tr}\left(D_{1} D_{2}^{t}\right)=-\frac{1}{2} \operatorname{tr}\left(D_{1} D_{2}\right)
$$

and its associated norm, $\|D\|=\langle D, D\rangle^{1 / 2}$.

The estimate we are interested in is the following analog of Bernstein's inequality for the derivative of a trigonometric polynomial.

THEOREM 4.1. If $p_{n} \in \mathscr{P}_{n}$ and $D \in \mathfrak{g}$, then

$$
\left\|D p_{n}\right\|_{\infty} \leqq n\|D\|\left\|p_{n}\right\|_{\infty} .
$$

Proof. We need to show that

$$
\left|D p_{n}(m)\right|=\left|(d / d t) p_{n}(\exp t D \cdot m)_{t=0}\right| \leqq n\|D\|\left\|p_{n}\right\|_{\infty} .
$$

We can reduce the problem to showing that $p_{n}(\exp t D \cdot m)$ is, as a function of $t$, the restriction to the real axis of an entire function of exponential type at most $n\|D\|$. Because once this is done the desired estimate follows from Bernstein's inequality for the derivatives of such functions [13, p. 208], since

$$
\sup _{t}\left|p_{n}(\exp t D \cdot m)\right| \leqq\left\|p_{n}\right\|_{\infty} .
$$

As a further reduction, we note that since $p_{n}$ is a polynomial of degree at most $n$ 
in $f_{1}, \ldots, f_{l}$, it will suffice to show that $f_{i}(\exp t D \cdot m)$ is the restriction to the real axis of an entire function of exponential type at most $\|D\|$.

We proceed as follows. By the standard spectral theory for skew-symmetric matrices we can find an orthogonal matrix $O$ and real numbers $\theta_{1}, \ldots, \theta_{[l / 2}$ such that

$O D O^{-1}=D_{1}=\operatorname{diag}\left(\operatorname{sk}\left(\theta_{1}\right), \operatorname{sk}\left(\theta_{2}\right), \ldots, \operatorname{sk}\left(\theta_{[l / 2]}\right)\right), \quad$ where sk $(\theta)=\left[\begin{array}{rr}0 & \theta \\ -\theta & 0\end{array}\right]$.

A computation shows that $\|D\|^{2}=-\frac{1}{2} \operatorname{tr}\left(D_{1}^{2}\right)=\sum \theta_{j}^{2}$. Hence $\|D\| \geqq \max \left\{\left|\theta_{j}\right|\right\}$. Now the entries of $\exp t D_{1}$ are the functions $\pm \cos t \theta_{j}, \pm \sin t \theta_{j}$, so the entries of $\exp t D=O^{-1} \exp t D_{1} O$ are just linear combinations of these sines and cosines. Thus we see that $f_{i}(\exp t D \cdot m)=\left(\exp t D \cdot m, e_{j}\right)$ is a linear combination of the functions $\cos t \theta_{j}, \sin t \theta_{j}$. Since these latter functions are entire functions of exponential type at most $\max \left\{\left|\theta_{j}\right|\right\}$ which is less than $\|D\|$, so is $f_{i}$ and we are done.

We shall refer to the estimate of this theorem as Bernstein's inequality (for homogeneous spaces).

5. Converse theorems on homogeneous spaces. In order to prove the converse to Corollary 2.2 and related theorems, we need to introduce a metric on $M$ which is connected with the action of $G$. We first define a Riemannian metric on $M$ as follows. Let $\mathfrak{h}$ be the Lie algebra of $H$ and let $\mathfrak{m}=\mathfrak{h}^{\perp}$ be the orthogonal complement of $\mathfrak{h}$ in $\mathfrak{g}$. Then $\mathfrak{m}$ is (isomorphic to) the tangent space to $M$ at $e_{1}$. Since the inner product on $\mathfrak{g}$ is invariant under the adjoint action of $G$ on $g$ we can define a Riemannian metric on $M$ by translating the restriction of $\langle\cdot, \cdot\rangle$ to $\mathfrak{m} \times \mathfrak{m}$ over $M$. Let $\rho$ be the arc-length metric induced by this Riemannian metric on each connected component of $M$. If $M$ is not connected we extend $\rho$ to all of $M$ by setting $\rho\left(m_{1}, m_{2}\right)$ $=1+d$ where $d$ is the diameter of the components of $M$ and $m_{1}, m_{2}$ are in distinct components of $M$.

By using properties of this metric and the Bernstein inequality we prove

LEMMA 5.1. If $p_{n} \in \mathscr{P}_{n}$ and $m_{1}, m_{2}$ are in the same component of $M$, then

$$
\left|p_{n}\left(m_{1}\right)-p_{n}\left(m_{2}\right)\right| \leqq \rho\left(m_{1}, m_{2}\right) n\left\|p_{n}\right\|_{\infty} .
$$

Proof. It follows from [10, p. 52] that there is a minimal geodesic from $m_{2}$ to $m_{1}$ of the form $t \mapsto \exp t D \cdot m_{2}, t \in[0,1]$, with $D \in \mathfrak{g}$ and $\|D\|=\rho\left(m_{1}, m_{2}\right)$. Thus

$$
\begin{aligned}
\left|p_{n}\left(m_{1}\right)-p_{n}\left(m_{2}\right)\right| & \leqq \int_{0}^{1}\left|(d / d t) p_{n}\left(\exp t D \cdot m_{2}\right)\right| d t \\
& =\int_{0}^{1}\left|D p_{n}\left(\exp t D \cdot m_{2}\right)\right| d t \\
& \leqq\left\|D p_{n}\right\|_{\infty} .
\end{aligned}
$$

Hence, by Bernstein's inequality we are done. 
This lemma and Bernstein's inequality make it possible to deduce various differentiability and smoothness properties of $f$ from knowledge about $E_{n}(f)$. The next two theorems are of this type and the proofs are quite like those for periodic functions on $\boldsymbol{R}$ (e.g., as presented in [5, pp. 58-62]), so we simply state the results.

THEOREM 5.2. There exists $C>0$ such that for $f \in C(M)$

$$
\left|f\left(m_{1}\right)-f\left(m_{2}\right)\right| \leqq C \rho\left(m_{1}, m_{2}\right) \Sigma^{\prime} E_{n}(f)
$$

where $\Sigma^{\prime}$ is the sum over $n \leqq 1 / \rho\left(m_{1}, m_{2}\right)$. (C=10 will suffice.)

To discuss differentiability properties we let $D^{k}=D_{1} D_{2} \cdots D_{k}, D_{i} \in \mathfrak{g}$, and set $\left\|D^{k}\right\|=\prod\left\|D_{i}\right\|$, then we have

THEOREM 5.3. If $\sum_{1}^{\infty} n^{k-1} E_{n}(f)<\infty$, then $f \in C^{k}(M)$. Moreover, there exists $C(k)>0$ such that

$$
E_{j}\left(D^{k} f\right) \leqq C(k)\left\|D^{k}\right\| \sum_{n>j / 2} n^{k-1} E_{n}(f) .
$$

$\left(C(k)=2^{2 k+1}\right.$ will suffice.) Also if $A$ is the right member of the above inequality when $j=1$, then $\left\|D^{k} f\right\|_{\infty} \leqq A+2\left\|D^{k}\right\| E_{0}(f)$.

We can use these two theorems to prove the Bernstein type of converse theorems characterizing the classes $C^{k,(\alpha)}(M), 0<\alpha<1$, in terms of $E_{n}(f)$. To do this we must connect the local differentiability and Lipschitz conditions of $\S 1$, with the global consequences of Theorems 5.2 and 5.3. This connection follows from

LEMMA 5.4. If $\varphi: B \rightarrow M$ is any local chart for $M$, then there exists $C>0$ such that $\rho(\varphi(x), \varphi(y)) \leqq C\|x-y\|$.

Proof. The lemma is proved by comparing Riemannian metrics on the tangent bundle to $\varphi(B)$. We have two such metrics, $\langle\cdot, \cdot\rangle$, induced from the inner product on $\mathfrak{m}$, and $\langle\cdot, \cdot\rangle^{\prime}$, induced from the flat Riemannian metric on $B$. If $\|\cdot\|_{m}$ and $\|\cdot\|_{m}^{\prime}$ are the associated norms at $m \in \varphi(B)$, then by compactness of $B$, there exists $C>0$ with $\|\cdot\|_{m} \leqq C\|\cdot\|_{m}^{\prime}$ for all $m \in \varphi(B)$. Thus if $\psi(t)=\varphi(t x+(1-t) y), t \in[0,1]$, is the "straight line" from $\varphi(y)$ to $\varphi(x)$ in the flat metric, we have

$$
\begin{aligned}
\rho(\varphi(x), \varphi(y)) & \leqq \int_{0}^{1}\left\|\frac{d \psi}{d t}\right\|_{\psi(t)} d t \\
& \leqq C \int_{0}^{1}\left\|\frac{d \psi}{d t}\right\|_{\psi(t)}^{\prime} d t=C\|x-y\| .
\end{aligned}
$$

We now have the converse of Corollary 2.2 in case $\alpha<1$.

THEOREM 5.5. Let $M$ be the orbit of some orthogonal G-action on $E$. Fix a norm on $C^{k,(\alpha)}(M)$. If $0<\alpha<1$, then there exist constants $B(k, \alpha)$ such that $E_{n}(f) \leqq A n^{-k-\alpha}$ implies $f \in C^{k,(\alpha)}(M)$ and $\|f\|_{k,(\alpha)} \leqq B(k, \alpha) A$. 
Proof. Theorems 5.2 and 5.3 show that $f \in C^{k}(M)$ and for some constants $C(k, \alpha)$,

$$
\left\|D^{j} f\right\|_{\infty} \leqq\left\|D^{j}\right\| C(k, \alpha) A, \quad j \leqq k
$$

and

$$
\left|D^{k} f\left(m_{1}\right)-D^{k} f\left(m_{2}\right)\right| \leqq C(k, \alpha)\left\|D^{k}\right\| A \rho^{\alpha}\left(m_{1}, m_{2}\right) .
$$

If $\varphi: B \rightarrow M$ is a chart then Lemma 5.4 and the last inequality imply that

$$
\left|D^{k} f \circ \varphi(x)-D^{k} f \circ \varphi(y)\right| \leqq C(k, \alpha, \varphi)\left\|D^{k}\right\| A\|x-y\|^{\alpha},
$$

or,

$$
\left|D^{k} f\right|_{(\alpha)} \leqq C(k, \alpha, \varphi)\left\|D^{k}\right\| A .
$$

Now since any partial derivative $\partial / \partial x^{\beta}$ can be written as a finite linear combination of operators $D^{j}, j \leqq|\beta|$, with $C^{\infty}$ coefficients these inequalities imply that $f \circ \varphi \in C^{k,(\alpha)}(B)$ and $\|f \circ \varphi\|_{k,(\alpha)} \leqq C^{\prime}(k, \alpha, \varphi) A$. If we cover $M$ by the finitely many charts used to define the norm in $C^{k,(\alpha)}(M)$ we have our result.

In case $\alpha=1$, the estimates of Theorems 5.2 and 5.3 will not show that $D^{k} f$ admits $\omega(t)=t$ as a modulus of continuity. However, for this case the analog of Zygmund's converse theorem on the circle is true for functions on homogeneous spaces.

THEOREM 5.6. There exists $C>0$ such that if $m_{+}, m, m_{-}$are equally spaced along a geodesic in $M$, then

$$
\left|f\left(m_{+}\right)-2 f(m)+f\left(m_{-}\right)\right| \leqq C \rho^{2}\left(m_{+}, m\right) \Sigma^{\prime}(n+1) E_{n}(f)
$$

where $\Sigma^{\prime}$ is the sum over $n \leqq 1 / \rho\left(m_{+}, m\right)$.

Corollary 5.7. There exist constants $B(k)$ such that $E_{n}(f) \leqq A n^{-k-1}$ implies $f \in C^{k}(M)$ and $\left|D^{k} f\left(m_{+}\right)-2 D^{k} f(m)+D^{k} f\left(m_{-}\right)\right| \leqq A B(k)\left\|D^{k}\right\| \rho\left(m_{+}, m\right)$.

The proofs are as in [5, pp. 59-61] once one has the obvious analog of Lemma 5.1 for second differences along geodesics.

We are in the strange situation of having proved a converse type of theorem without knowing the corresponding direct theorem. This results from the fact that we have not been able to prove that the local second order modulus of continuity is invariant under coordinate changes. However, for those homogeneous spaces for which we know more constructive proofs of the direct theorems, in particular, for all spheres, and projective spaces over the reals, complexes and quaternions, we have proved in [12] the direct theorems corresponding to Corollary 5.7.

6. Remarks on applications and earlier results. The results of $\$ 2$ have several applications to problems of Fourier analysis on the compact homogeneous spaces considered in $\$ \$ 4$ and 5 analogous to the applications of the results on tori to 
multiple Fourier series. In particular, they can be used to generalize the Bernstein theorem on absolute convergence of Fourier series for functions in $C^{0,(1 / 2+\varepsilon)}\left(S^{1}\right)$ and the Dini-Lipschitz theorem on uniform convergence of Fourier series for functions admitting $\omega(t)=o(1 / \log (1 / t))$ as a modulus of continuity. Some of these are in [11] and they will all appear in some articles we are currently preparing.

The Jackson type estimates of Theorem 2.1 have previously been proved for certain compact manifolds in addition to the cases of cubes and tori. For instance, Gronwall [3] handled the case $M=S^{2}, k=0$, while much later his work was extended to all $k$ by Kušnirenko [4] and Džafarov [1]. Also Newman and Shapiro [9] considered the case $M=S^{n}, k=0$, and we have recently extended their approach to all $k$ [12]. For $M$ a unitary group Sun Kung [2] obtained results similar to Corollary 2.2 for $k=0$.

Converse theorems similar to Theorems 5.2, 5.3 and 5.5 were proved for the tori by M. F. Timan, see [8, pp. 350-358]. The only other compact manifold for which we have seen such theorems is $S^{2}$ in Kušnirenko [4] and Džafarov [1].

\section{REFERENCES}

1. A. S. Džafarov, On the order of the best approximations of the functions continuous on the unit sphere by means of finite spherical sums, Proc. Second All-Union Conference (Baku, 1962), Studies Contemporary Problems Constructive Theory of Functions, Izdat. Akad. Nauk Azerbaǐdžan. SSR, Baku, 1965, pp. 46-52. (Russian) MR 33 \#6227.

2. Sun Kung, Fourier analysis on unitary groups. IV: On the Peter-Weyl theorem, Acta Math. Sinica 13 (1963), 323-331 = Chinese Math.-Acta 4 (1964), 351-359. MR 31 \#4861.

3. T. H. Gronwall, On the degree of convergence of Laplace series, Trans. Amer. Math. Soc. 15 (1914), 1-30.

4. G. G. Kušnirenko, The approximation of functions defined on the unit sphere by finite spherical sums, Naučn. Dokl. Vyš̌. Skoly Fiz.-Mat. Nauki 1958, no. 4, 47-53. (Russian) MR 26 \#530.

5. G. G. Lorentz, Approximation of functions, Holt, Rinehart and Winston, New York, 1966. MR 35 \#4642.

6. G. D. Mostow, Equivariant embeddings in Euclidean space, Ann. of Math. (2) 65 (1957), 432-446. MR 19, 291.

7. J. Nash, Real algebraic manifolds, Ann. of Math. (2) 56 (1952), 405-421. MR 14, 403.

8. D. J. Newman and L. Raymon, A class of curves on which polynomials approximate efficiently, Proc. Amer. Math. Soc. 19 (1968), 595-599. MR 37 \#653.

9. D. J. Newman and H. S. Shapiro, Jackson's theorem in higher dimensions (With discussion), Proc. Conference on Approximation Theory (Oberwolfach, 1963), Birkhäuser, Basel, 1964, pp. 208-219. MR 32 \#310.

10. K. Nomizu, Invariant affine connections on homogeneous spaces, Amer. J. Math. 76 (1954), 33-65. MR 15, 468.

11. D. L. Ragozin, Approximation theory on compact manifolds and Lie groups, with applications to harmonic analysis, PhD. Thesis, Harvard University, Cambridge, Mass., 1967.

12. - Constructive polynomial approximation on spheres and projective spaces, Trans. Amer. Math. Soc. (to appear).

13. A. F. Timan, Theory of approximation of functions of a real variable, Fizmatgiz, Moscow, 
1960; English transl., Internat. Series of Monographs in Pure and Appl. Math., vol. 34, Macmillan, New York, 1963. MR 22 \#8257; MR 33 \#465.

14. H. Whitney, Elementary structure of real algebraic varieties, Ann. of Math. (2) 66 (1957), 545-556. MR 20 \#2342.

15. O. Zariski and P. Samuel, Commutative algebra. Vol. II, The University Series in Higher Math., Van Nostrand, Princeton, N. J., 1960. MR 22 \#11006.

Massachusetts Institute of Technology,

CAmbridge, Massachusetts 02139

UNIVERSITY OF WASHINGTON,

Seattle, Washington 98105 